\title{
Synergistic Effect of Garcinia Mangostana Combined With Chlorhexidine on Acanthamoeba Triangularis Trophozoites and Cysts
}

\section{Suthinee Sangkanu}

School of Allied Health Sciences, Southeast Asia Water Team (SEA Water Team) and World Union for Herbal Drug Discovery (WUHeDD), and Research Excellence Center for Innovation and Health Products (RECIHP), Walailak University, Nakhon Si Thammarat, Thailand.

\section{Watcharapong Mitsuwan}

School of Allied Health Sciences, Southeast Asia Water Team (SEA Water Team) and World Union for Herbal Drug Discovery (WUHeDD), and Research Excellence Center for Innovation and Health Products (RECIHP), Walailak University, Nakhon Si Thammarat, Thailand.

\section{Wilawan Mahabusarakam}

Graduate School, Prince of Songkla University, Songkhla, Thailand.

\section{Tajudeen 0. Jimoh}

Department of Pharmacognosy and Pharmaceutical Botany, Faculty of Pharmaceutical Sciences, Chulalongkorn University, Bangkok, Thailand.

\section{Polrat Wilairatana}

Department of Clinical Tropical Medicine, Faculty of Tropical Medicine, Mahidol University, Bangkok, Thailand.

\section{Ana Paula Girol}

Department of Biology, Faculty of Sciences, São Paulo State University, São Paulo, Brazil.

\section{Ajoy K. Verma}

Department of Microbiology, National Institute of Tuberculosis \& Respiratory Diseases (NITRD), New Delhi, India

\section{Maria de Lourdes Pereira}

Department of Medical Sciences \& CICECO-Aveiro Institute of Materials, University of Aveiro, Aveiro, Portugal

\section{Mohammed Rahmatullah}

Department of Biotechnology \& Genetic Engineering, University of Development Alternative Lalmatia, Dhaka, Bangladesh.

\section{Christophe Wiart}

School of Pharmacy, University of Nottingham Malaysia Campus, Selangor, Malaysia

\section{Abolghasem Siyadatpanah}

Ferdows School of Paramedical and Health, Birjand University of Medical Sciences, Birjand, Iran. 


\section{Veeranoot Nissapatorn ( $\nabla$ nissapat@gmail.com )}

School of Allied Health Sciences, Southeast Asia Water Team (SEA Water Team) and World Union for Herbal Drug Discovery (WUHeDD), and Research Excellence Center for Innovation and Health Products (RECIHP), Walailak University, Nakhon Si Thammarat, Thailand.

\section{Research}

Keywords: Garcinia mangostana pericarp extract, Anti-Acanthamoeba activity, Acanthamoeba triangularis, Synergistic effect

Posted Date: August 11th, 2020

DOI: https://doi.org/10.21203/rs.3.rs-55506/v1

License: (c) (i) This work is licensed under a Creative Commons Attribution 4.0 International License. Read Full License 


\section{Abstract}

Background: Various parts of Garcinia mangostana Linn, including its pericarp have been traditionally used to treat different types of diseases. This study was carried out to determine the anti-Acanthamoeba activity of G. mangostana pericarp extract against Acanthamoeba triangularis.

Methods: The G. mangostana ethanolic pericarp extract was screened for the anti-Acanthamoeba activity and determined its minimal inhibition concentrations (MICs) by the microdilution method. Then, the timekill kinetic assay of the extract was determined. The synergistic effect of $G$. mangostana extract and chlorhexidine was performed using the checkerboard method. Parasite morphology was detected by scanning electron microscopy (SEM).

Results: The MICs of extract were assessed on trophozoites and cysts with 250 and $4000 \mu \mathrm{g} / \mathrm{mL}$, respectively. More so, at 2xMIC of extract exhibited inhibitory activity against trophozoites and cyst of $A$. triangularis for up to 7 days. Checkerboard assays showed synergistic activity of extract (500$1000 \mu \mathrm{g} / \mathrm{mL}$ ) plus chlorhexidine (3.90-15.62 $\mu \mathrm{g} / \mathrm{mL}$ ) at a fractional inhibitory concentration index (FICl) of $0.18-0.37$. The lowest $\mathrm{FICl}(0.18)$ displayed good synergism resulting in up to 16 -fold reduction of drug MIC and reducing to 8 -fold of extract MIC. The viability of cysts decreased to $12.28 \pm 3.03 \mathrm{cell} / \mathrm{mL}$. FICI interpretation equal to 1 is considered an additive effect on Acanthamoeba trophpzoites. The SEM results clearly showed that Acanthamoeba cells treated with a single drug of chlorhexidine and its combination with G. mangostana extract caused cell membrane damage and irregular cell shapes comparing with the control.

Conclusion: A good combinatorial relationship displayed by G. mangostana extract and chlorhexidine suggest a more reliable evidenced based therapeutic strategy. Therefore, this approach is promising and could be employed as an alternative treatment method for the management of Acanthamoeba infection.

\section{Introduction}

Acanthamoeba is an opportunistic pathogen that is isolated from diverse natural environments [1]. This organism exists in two main forms: the trophozoite, which is the invasive stage; and the cysts, which is the highly resistant stage in a very harsh environment [2]. Acanthamoeba spp. have been divided into three groups (I, II, and III) based on characteristics of cyst such as size and shape. However, Acanthamoeba spp. were then classified into 20 different genotypes (T1-T20 Genotype) based on $18 \mathrm{~S}$ rRNA gene sequencing. Genotype T4 is the most commonly isolated in both clinical and environmental samples, followed by genotype T3 and T5. In addition, the T4 genotype is the most virulent because it has a higher potential of binding to host cells than other genotypes [3].

Acanthamoeba spp. are the causative agents of amebic keratitis (AK) and granulomatous amebic encephalitis (GAE). AK can cause permanent vision loss or blindness [4]. The rate of infectious keratitis is becoming alarming in recent time which is an issue that could be linked to a sudden increase in the population of contact lens wearers [2]. Similarly, Acanthamoeba encysts sprawled deep within the corneal 
stroma [5], as such, the cyst wall becomes impermeable to the existing drugs and this has reduced further research in the areas of drug formulations and designed for this organism.

Garcinia mangostana Linn is generally known as mangosteen and belongs to the family Clusiaceae. It is a tropical plant, widely grown in Southeast Asia [6]. The pericarps of this fruit have been used for traditional medicine in treating many diseases [7]. The xanthone group, enriched in G. mangostana extract exhibited anti-oxidant properties, anti-bacterial activity, anti-diabetic activity, anti-tumour, antiinflammatory, anti-allergenic, anti-malarial activity, anti-bacteria, cytoprotective and anti-cancer properties [8]. In 2017, Cantos and Lee [9] reported the ovicidal activity of peel and seed extracts from $G$.

mangostana against Ascaris suum. Next year, Canton and Jagunap [10] found that the extract from pericarp had better-inhibited ova fertilization than mebendazole.

Having searched through all available information on this plant, we discovered to our surprise that there is no single report on the anti-amoebic activity of G. mangostana extract against Acanthamoeba spp. Hence, our study sought to investigate the effective concentration of $G$. mangostana extract for inhibiting Acanthamoeba spp. and demonstrate synergistic effects of extract from $G$. mangostsana pericarp and chlorhexidine on anti-amoebic activity against $A$. triangularis.

\section{Materials And Methods}

\section{Preparation of plant extracts}

Dried mangosteen pericarp powder was extracted with ethanol. $50 \mathrm{~g}$ of powdered mangosteen pericarp was soaked in $200 \mathrm{~mL}$ of ethanol for 7 days. The extract was filtered through Whatman No. 1 using vacuum and pressure pump. The solution was evaporated to dryness under reduced pressure using a rotary evaporator to obtain a G. mangostana extract. The extract was dissolved in $100 \%$ DMSO and stored at $-20^{\circ} \mathrm{C}$ until use.

\section{Cultivation of A. triangularis}

A. triangularis WU19001, a strain from the recreational reservoir at Walailak University, Nakhon Si Thammarat Thailand was used in this study [11]. The parasite was grown in PYG medium $(0.75 \%(\mathrm{w} / \mathrm{v})$ proteose peptone, $0.75 \%(\mathrm{w} / \mathrm{v})$ yeast extract and $1.5 \%(\mathrm{w} / \mathrm{v})$ glucose). The trophozoites were observed after 48-72 hours of incubation at room temperature and were cultured in this medium for 1 week in order to obtain mature cysts. Trophozoites cells and cysts were centrifuged at $4000 \mathrm{rpm}$ for 5 minutes and re-suspended in fresh PYG thereafter. The viability was investigated using $0.1 \%$ trypan blue assay and adjused to $2 \times 10^{5}$ cells $/ \mathrm{mL}$.

\section{In vitro effect of plant extract against A. triangularis}


One hundred microliters of trophozoites and cysts suspension $\left(2 \times 10^{5}\right.$ cells $\left./ \mathrm{mL}\right)$ were added to 96-well microplates that contained $100 \mu$ of plant extract (final concentrations of $4000 \mu \mathrm{g} / \mathrm{mL}$ ). Chlorhexidine and $1 \%$ DMSO were used as positive and negative controls respectively. Viability was determined using $0.1 \%$ trypan blue that was obtained by manual count with inverted microscopy. The relative percentage of parasite viability was defined as (mean of the treated parasite/mean of the control) $\times 100$.

\section{Determination of minimal inhibitory concentration (MIC) of G. mangostana extract against A. triangularis}

The minimum inhibitory concentration (MIC) for plant extract was determined using microtiter broth dilution method [11]. Plant extract was diluted to give a final concentration of $4000,2000,1000,500,250$, $125,62.5 \mu \mathrm{g} / \mathrm{mL}$ into 96 -well microplate. Then $100 \mu \mathrm{l}$ of $2 \times 10^{5} \mathrm{cells} / \mathrm{mL}$ of trophozoites and cysts were inoculated in each well. Chlorhexidine and 1\% DMSO were included as positive and negative control, respectively. The plates were incubated at room temperature for 24 hours. The MIC value of the extract was defined as the lowest concentration that inhibited $>90 \%$ of growth, when compared with the negative control.

\section{Time-kill Assay}

Time-kill kinetics of the ethanolic extract of $G$. mangostana was carried out following the procedure as described [12] with modification. $100 \mu \mathrm{L}$ of $2 \times 10^{5}$ cells $/ \mathrm{mL}$ of trophozoites and cysts were inoculated into microcentrifuge tube containing PYG with extract at final concentration $2 \times \mathrm{MIC}, \mathrm{MIC}, 0.5 \times \mathrm{MIC}$, and $0.25 \times$ MIC and incubated at room temperature for 1 week. The aliquots of $A$. triangularis were removed and counted at 24 hours intervals using hemocytometer. $A$. triangularis treated with $1 \%$ DMSO was used as a control.

\section{Combination of G. mangostana extract with chlorhexidine in targeting trophozoites and cysts of A. triangularis}

Checkerboard assay method was used to evaluate the interaction between $G$. mangostana extract and chlorhexidine against $A$. triangularis. The checkerboard method [13] used was the microdilution assay performed in a 96-well plate with final volume of $200 \mu \mathrm{L}$. G. mangostana extract and chlorhexidine were diluted with PYG to obtain 4 times to its final concentrations of 1/16 MIC, 1/8 MIC, 1/4 MIC, 1/2 MIC and MIC. Fifty $\mu \mathrm{l}$ of extract and chlorhexidine were transferred to a microtiter plate containing $100 \mu \mathrm{L}$ of $2 \times$ $10^{5} \mathrm{cells} / \mathrm{mL}$ of trophozoites and cysts. The plates were incubated at room temperature for 24 hours. The viability of the extract was defined as the lowest concentration that inhibited $>90 \%$ of growth, when compared with the negative control. The assessment of results was defined as Fractional Inhibitory Concentration Index (FICl) that was calculated utilizing the following 
$\mathrm{FICl}$ of combination $=\mathrm{FIC} \mathrm{A}+\mathrm{FIC} \mathrm{B}$

FIC A = MIC of chlorhexidine in combination / MIC of chlorhexidine alone

FIC B $=$ MIC of extract in combination / MIC of extract alone.

The combination was considered synergistic for $\Sigma F I C \leq 0.5$, additive for $0.5<\Sigma F I C \leq 1$, indifferent for $1<$ $\Sigma F I C<4$, and antagonistic for $\Sigma F I C \geq 4$, according to EUCAST definition.

\section{Scanning Electron Microscopic (sem) Study}

Acanthamoeba trophozoites and cysts were treated with chlorhexidine and G. mangostana extract. After incubation, cells were collected by centrifugation at $4000 \mathrm{rpm}$ for 5 minutes and re-suspended in phosphate buffer saline (PBS). Cells in DMSO (1\%) were used as negative controls. Samples were fixed with $2.5 \%$ glutaradehyde overnight. The sample was further dehydrated with graded alcohol series $(20 \%$, $40 \%, 60 \%, 80 \%, 90 \%$, and $100 \%$ ethanol), mounted on aluminum stubs, and allowed to dry using a critical point dryer. The samples were then coated with gold particles and the morphology of Acanthamoeba trophozoites and cysts after treatment was subsequently examined under SEM (SEM-Zeiss, Munich, Germany) at the Center for Scientific and Technological Equipment, Walailak University, Nakhon Si Thammarat, Thailand.

\section{Statistical Analysis}

The experiments were performed in triplicate. All data were recorded and entered using the statistical package software (SPSS Inc. Chicago, IL, USA). The data were expressed as mean \pm SD. Statistical analysis was analyzed by the two-tailed unpaired Student's t-test. In all analyzes, $\mathrm{P}<0.05$ was considered statistically significant.

\section{Results}

1. G. mangostana extract exhibited anti-Acanthamoeba activity against A. triangularis

Anti-Acanthamoeba activity was performed to observe the effects of the G. mangostana extract on viability of $A$. triangularis. The extract showed significant reduction in trophozoites viability as compared to the control $(P<0.05)$ (Fig. 1a). At $250-2000 \mu \mathrm{g} / \mathrm{mL}$ of extract reduced the trophozoites viability to 5.8$8.8 \%$. Furthermore, the viability percentages of cysts decreased to $10.7-13.8 \%$ after incubated with $2000-4000 \mu \mathrm{g} / \mathrm{mL}$ of extract $(P<0.05)$ (Fig. 1b).

2. Minimum inhibitory concentration (MIC) of G. mangostana extract against $\mathrm{A}$. triangularis

Extract of $G$. mangostana pericarp was determined for its anti-Acanthamoeba potential by MIC using microtiter dilution broth method. As shown in Table 1, the MICs of mangosteen ethanol extract against $A$. 
triangularis trophozoites and cysts were 250 and $4000 \mu \mathrm{g} / \mathrm{mL}$, respectively. The MIC values of chlorhexidine against trophozoites and cysts were 7.81 and $62.5 \mu \mathrm{g} / \mathrm{mL}$, respectively.

Table 1

Minimal inhibitory concentration (MIC) of $G$. mangostana extract and chlorhexidine against $A$. triangularis trophozoites and cysts.

\begin{tabular}{|lll|}
\hline Antimicrobial agents & \multicolumn{2}{l|}{ MIC $(\mu \mathrm{g} / \mathrm{mL})$} \\
\cline { 2 - 3 } & Trophozoites & Cysts \\
\hline G. mangostana extract & 250 & 4000 \\
Chlorhexidine & 7.81 & 62.5 \\
\hline
\end{tabular}

3. Time-kill kinetic study of G. mangostana extract against A. triangularis trophozoites and cysts

The number of trophozoites reached from $2.0 \times 10^{5}$ cells $/ \mathrm{mL}$ to $1.3 \times 10^{6}$ cells $/ \mathrm{mL}$ within 7 days. Similarly, population of cysts exhibited an increase number from $2.0 \times 10^{5}$ cells $/ \mathrm{mL}$ to $9.7 \times 10^{6} \mathrm{cells} / \mathrm{mL}$ (Fig. 2a and b). All concentrations of G. mangostana extract inhibited the growth of Acanthamoeba trophozoites and cysts when compared with the control. At $2 \times$ MIC of the extract displayed strongest inhibitory activity in both forms of Acanthamoeba. After 7 days, the number of viable trophozoites were detected at $2.1 \times 10^{5}$ cells $/ \mathrm{mL}$ in the treatment with $2 \times \mathrm{MIC}$ extract (Fig. 2a), while the viable cysts was $1.3 \times 10^{5}$ cells $/ \mathrm{mL}$ (Fig. $2 \mathrm{~b}$ ).

4. Synergistic effects of G. mangostana extract in combination with chlorhexidine against A. triangularis by the checkerboard assay

Evaluation of synergistic effects between the extract and drug was determined in this study. Figure. 3 shows the viable trophozoites in combination test. The lowest FIC values (0.5) for each agent reduced viability of trophozoites to $12.72 \times 10^{5}$ cells $/ \mathrm{mL}$. FICl interpretation equal to 1 is to be considered an additive effect (Table 2). G. mangostana extract showed synergistic $(\mathrm{FICl}<0.5)$ activity with chlorhexidine against $A$. triangularis cysts. Set of combinations of extract showed $\mathrm{FICl}$ ranged from 0.18 to 0.37 (Table 2). The lowest $\mathrm{FICl}(0.18)$ displayed good synergy resulting in up to16-fold reduction of drug MIC and a reduction to 8 -fold of extract MIC. The viability of cysts decreased to $12.28 \pm 3.03$ cells $/ \mathrm{mL}$ (Fig. 4).

\section{SEM analysis of G. mangostana extract effects on A. triangularis}

The alterations due to the action of the extract was confirmed by SEM micrograph are shown in Figs. 5 and 6. Control trophozoites (Figs. 5a, e and i) treated with 1\% DMSO exhibited normal cells with acanthopodia. Treated trophozoites with $4 \times$ MIC of chlorhexidine showed flat cells and smooth surface which is a feature that could be related to a total destruction of acanthopodia (Figs. $5 b, f$, and j). In treatment with $4 \times \mathrm{MIC}$ of $G$. mangostana extract, the damage cells were observed as flat and smooth surface similar to $4 \times$ MIC of chlorhexidine treated (Figs. $5 \mathrm{c}, \mathrm{g}$, and k). Trophozoites treated with the 
combination of chlorhexidine/G. mangostana extract at FICl equal 1, SEM showed rounded morphology with pores on cell surface (Figs. $5 \mathrm{~d}, \mathrm{~h}$, and I). Also, the cysts micrographs of control cells showed intact cell with oval and smooth surface as showed in Figs. 6a, e and i. The oval cysts of Acanthamoeba were flat, irregularly shaped causing collapse of the ectocyst walls after treatment with $4 \times$ MIC of chlorhexidine (Figs. 6b, f, and j), $4 \times$ MIC of G. mangostana extract (Figs. 6c, g, and k) and the combination of chlorhexidine/ G. mangostana extract at $\mathrm{FICl}$ equal 0.18 (Figs. 6d, h, and I).

Table 2 Fractional inhibitory concentration index (FICl) of the combination test of $G$. mangostana extract with chlorhexidine in

targeting trophozoites and cysts of $A$. triangularis

\begin{tabular}{|c|c|c|c|c|c|c|}
\hline \multirow{2}{*}{$\begin{array}{l}\text { A. triangularis } \\
\text { infective } \\
\text { stages }\end{array}$} & \multirow{2}{*}{$\begin{array}{l}\text { MIC } \\
\text { chlorhexidine } \\
\text { alone ( } \mu \mathrm{g} / \mathrm{ml})\end{array}$} & \multirow{2}{*}{$\begin{array}{l}\text { MIC extract } \\
\text { alone } \\
(\mu \mathrm{g} / \mathrm{ml})\end{array}$} & \multicolumn{2}{|c|}{$\begin{array}{l}\text { MIC in combination } \\
(\mu \mathrm{g} / \mathrm{ml})\end{array}$} & \multirow[t]{2}{*}{$\mathrm{FICl}^{\mathrm{a}}$} & \multirow[t]{2}{*}{ Effect } \\
\hline & & & chlorhexidine & extract & & \\
\hline Trophozoties & 7.81 & 250 & 3.90 & 125 & 1 & Additivity \\
\hline \multirow[t]{5}{*}{ Cysts } & 62.5 & 4000 & 3.90 & 500 & 0.18 & Synergistic \\
\hline & & & 7.81 & 500 & 0.24 & Synergistic \\
\hline & & & 15.62 & 500 & 0.36 & Synergistic \\
\hline & & & 3.90 & 1000 & 0.31 & Synergistic \\
\hline & & & 7.81 & 1000 & 0.37 & Synergistic \\
\hline
\end{tabular}

a The combination was considered synergistic for $\Sigma F I C \leq 0.5$, additive for $0.5<\Sigma F I C \leq 1$, indifferent for 1 $<\Sigma F I C<4$, and antagonistic for $\Sigma F I C \geq 4$.

\section{Discussion}

Herb-drug interaction may offer potential health promoting effect by augmenting drug efficacy or diminishing potential side effects. As such, we have presented an effective treatment of Acanthamoeba infections with herb-drug based combination strategy in this study. Also, we have highlighted the potential of anti-Acanthamoeba activity of $G$. mangostana ethanol extract. It is a natural source that provides active components of G. mangostana also known as the "queen of tropical fruits". The $G$. mangostana extracts have anti-oxidant, anti-tumoral, anti-allergic, anti-inflammatory, anti-bacterial and 
anti-viral activities [8]. A few studies have reported the effects of $G$. mangostana extract on anti-parasitic activity but there was no report on its Acanthamoebic effects.

A. triangularis WU19001 was a selected strain parasite used in this study. A. triaugularis was characterized in group II (type 4) typically a wrinkled ectocyst and an endocyst which could be stellate, polygonal, triangular, or oval [14]. A. triangularis was originally isolated from the human feces documented in France [15]. In 2008, the first case of keratitis caused by A. triangularis had been reported in Korea [16]. Also, six isolates of $A$. triangularis had also been reported from cases with contaminated contact lens in southeastern Korea. In support of those previous findings, our study revealed that $G$. mangostana extract from pericarp exhibits anti-Acanthamoeba activity against $A$. triangularis. The MIC values of G. mangostana extract were 250 and $4000 \mu \mathrm{g} / \mathrm{ml}$ against Acanthomoeba trophozoites and cysts, respectively (Table 1). Our finding is in agreement with earlier reported studies that found the compounds of $G$. mangostana extract with anti-parasitic activities. In literature, xanthones have been identified as the main compound of $G$. mangostana extract. Of this, xanthones have been isolated from pericarp, whole fruit, leaves and bark [3]. $a$ - and $y$-mangostin are the most abundant xanthones found in the pericarp of mangosteen fruit. Other xanthones in mangosteen pericarp include $\beta$-mangostin, gartanin, 8-deoxygartanin, garcinones $A, B, C, D$ and E, mangostinone, 9-hydroxycalabaxanthone and isomangostin, among others $[3,17]$. Interestingly, the a-mangostin compound of $G$. mangostana has been reported with $\mathrm{IC}_{50}$ value of $17 \mu \mathrm{M}$ against Plasmodium falciparum [18]. Another study showed that a-mangostin and $\delta$-mangostin exhibited anti-malarial activity against $P$. falciparum chloroquine-resistant strain with $\mathrm{IC}_{50}$ value 0.2 and $121.2 \mu \mathrm{M}$, respectively [19]. Furthermore, a previous study has reported the use of $G$. mangostana pericarp in the management of amoebic dysentery [8]. Xanthones block the polymerization process, and accumulation of soluble heme-drug complexes increases the osmotic pressure in the vacuole, causing its lysis on trophozoite stage [20]. The synthetic Caged Garcinia xanthones (CGXs) exhibit anti-malarial activity against $P$. falciparum with low toxicity. It was reported that CGXs treatment affect malaria parasites relating to morphological changes, significant reduction of parasitemia (the percentage of infected red blood cells), and aberrant mitochondrial fragmentation [21]. Benzophenone (isoxanthochymol) was isolated from the roots of $G$. polyantha which showed strong chemosuppressive activity of parasitic growth found in $P$. falciparum [22]. The benzophenones (guttiferone $\mathrm{E}$, isoxanthochymol, and guttiferone $\mathrm{H}$ ) isolated from $\mathrm{G}$. xanthochymus exhibited antiplasmodial activity with $\mathrm{IC}_{50}$ values in the range of $4.71-11.40 \mu \mathrm{M}$ [23]. The tetraprenylated benzophenone 7-epiclusianone (7-epi) was isolated from the fruits of $G$. brasiliensis showed effective against Schistosoma mansoni [24]. In addition, biflavanones (GB-1a, GB-1, and GB-2), another active compounds, were isolated from $G$. kola. These three biflavanones displayed the potent inhibitory activity in vitro against $P$. falciparum proliferation and also antimalarial potency through oral administration in mice infected with $P$. berghei without signs of acute toxicity [25]. As kolaviron (KV), a biflavonoid fraction from $G$. kola seeds that has also been reported to exhibit anti-malarial activities in P. berghei-infected mice [26]. The purified bioflavonoid of the fruit pericarp of $G$. brasiliensis, showed significant activity as inhibitors of Leishmania's proteases, with mean $( \pm S D) I_{50}$ values of $15.0 \pm 1.3 \mu \mathrm{g} / \mathrm{mL}$ [27]. Interestingly, the effect of xanthones has also been increasingly reported on targeting the bacterial membrane as a 
result of rapid bactericidal action led to reducing resistance process and mutational possibility [28]. Therefore, xanthones, biflavanones, and/or benzophenones may play a major role on the effect against A. triangularis in this study, however, it further suggests for more comprehensive studies to evaluate the mechanism of action on these compounds against $A$. triaugularis.

In fact, the eradication of Acanthamoeba infection seems impossible due to highly resistance of the cysts to anti-amoebic drugs. Therefore, we also investigated the effective concentration of $G$. mangostana extract against $A$. triangualris trophozoite and cyst stages. Our results interestingly showed that $G$. mangostana extract can inhibit $A$. triangularis in a longer incubation period in PYG medium. It's worthy to note that the number of Acanthamoeba trophozoite and cysts were reduced significantly $(P<0.05)$ in the presence of $G$. mangostana extract (MIC and $2 \times$ MIC final concentrations). To our observation, the $G$. mangostana concentrations was found to have the highest growth-inhibitory for trophozoites at day 3 and cysts at day 2 (Figs. 2a and 2b) though there are few remaining A. triangularis survived due to diminishing the effect of Garcinia extract on the days after. To the best of our knowledge, we have shown the first potentially report of $G$. mangostana extract in its capacity to significantly inhibit Acanthamoeba cysts in an enrichment medium up to one week. This study have also shown the survival rate of $A$. triangularis ( $<10 \%$ in trophozoites and $<15 \%$ in cysts) within the same period. Based on these results, it should therefore be better considered a 1-week than 3 days [29] as a general protocol for observation on the growth inhibitory effect of plant extract against $A$. triangularis. It is also very encouraging to explore the results shown that $G$. mangostana extract of $2 \times$ MIC remarkably inhibited the growth of both trophozoites and cysts up to 7 days. Our finding is demonstrated similarly to a previous report on the growth inhibition of $A$. culbertsoni and $A$. castellanii incubated in the nonpolar extract of Gastrochilus panduratum from day 2 to day 7 [12]. Overall, this study therefore suggests that $G$. mangostana extract is a promising plant that shows the remarkable effect against $A$. triangularis infections.

Chlorhexidine is the mainstay drug of choice for Acanthamoeba keratitis and encephalitis due to it is active against both cysts and trophozoites [5]. In this study, a single drug chlorhexidine was therefore used against $A$. triangularis and successfully exhibited inhibitory activity on trophozoites and cysts with MIC values of 7.81 and $62.5 \mu \mathrm{g} / \mathrm{mL}$ (Table 1), respectively. Our finding is in consistent with an earlier report on chlorhexidine used against $A$. triangularis exhibited amoebicidal and cysticidal properties at $200 \mu \mathrm{g} / \mathrm{mL}$ (0.02\%) [30]. While, a recent study [3] evaluated the efficacy of miltefosine, another orphan drug for the treatment of keratitis and encephalitis, against Acanthamoeba spp. cysts of genotypes T3, T4 and T5. Interestingly, the minimal cysticidal concentration (MCC) of $38.72 \mathrm{mM}$ and $77.44 \mathrm{mM}$ of miltefosine showed the effect against Acanthamoeba genotypes T4/T5 and T3, respectively. This suggests for further study to evaluate the effect of miltefosine against $A$. triangularis. In a matter of fact, a single drug used for treating infectious diseases includes this parasitic infection not only causes side effects, long-term use, costly, and drug resistance. Therefore, the combination approach is constantly introduced to encounter those pitfalls. Chlorhexidine has frequently been used in combination with aromatic diamidines [5], aminoglycosides, imidazoles and polyene [31], however these chemicals show side effects on keratocytes found in cases of human keratitis [32]. Recently, the finding of novel compounds with anti-Acanthamoeba activity of plant and herbs has been very encouraging to evaluate a 
source of new molecules with anti-Acanthamoeba effects. At the same time, there is no data on the use of synergistic interaction between plant extract and synthetic drug against Acanthamoeba infection till date. To support this, our study also revealed an additive effect of a combination between $G$.

mangostana extract and chlorhexidine against Acanthamoeba trophozoites (viable trophozoites $<90 \%$ )

(Fig. 3). An additive effect occurs when substance added together enhances or improves the efficacies but not to the extent of synergic interaction [33]. Our better results were obtained when $G$. mangostana extract was combined with chlorhexidine to produce a synergistic effect against $A$. triangularis cysts. The $\mathrm{FICl}$ values demonstrated the synergy for concentration of 3.90 to $15.62 \mu \mathrm{g} / \mathrm{mL}$ of chlorhexidine and 500 to $1000 \mu \mathrm{g} / \mathrm{mL}$ of G. mangostana extract as shown the viability of less than $10 \%$ (Fig. 4). It is interestingly found that the concentration of chlorhexidine can be 4-16 times lower in the presence of $G$. managostana extract but presenting the effects against cysts while reducing its toxicity. This finding is in line with a previous study demonstrated a synergistic effect against $A$. polyphaga from a combination of chlorhexidine digluconate (CLX) and carbosilane dendrimers containing ammonium or guanidine moieties [34]. From this study, it appears to be a promising combined agents to fight against the infection and especially resistance pattern of Acanthamoeba spp. in the future.

The mode of action considered in this study was confirmed by scanning electron microscopy (SEM) as shown in Figs. 5 and 6. Treated trophozoites showed similar flat cells and smooth surface as a result of total destruction of acanthopodia in the presence of G. mangostana extract and chlorhexidine. In combination, morphology of trophozoites were rounded and being observed of the presence of pores on its surface. Chlorhexidine is positively charged and ionic with the negatively charged plasma membrane of the parasite, resulting in membrane structure that gives rise to permeability modulation, ionic leakage, and cytoplasmic disruptions causing cellular damage and cell death [35-36]. The control cysts showed regular morphological characteristics. Overall, $A$. triangularis cysts were flat and morphological deformity (irregular in shape and size) as a result on destruction of the ectocyst walls after treatment given with $G$. mangostana extract, chlorhexidine and in combination of chlorhexidine/G. mangostana extract.

\section{Conclusion}

In line with the data obtained from this study, G. mangostana ethanolic extract from pericarp possesses anti-Acanthamoeba activity against both trophozoites and cysts. Moreover, it is clear that we have presented for the first time, an anti-Acanthamoeba activity with emphasis on herb-drug combinatorial approach. This study therefore recommends further research in this area as it could provide an alternative treatment method for the management of Acanthamoeba infections.

\section{Declarations}

\section{Acknowledgement}

We highly appreciate for the support of The Royal Patronage of Her Royal Highness Princess Maha Chakri Sirindhorn - Walailak University Botanic Garden, Nakhon Si Thammarat, Thailand. We are also 
grateful to the Research Institute of Health Science, Walailak University for the laboratory facilities.

\section{Ethics approval and consent to participate}

Not applicable

\section{Consent for publication}

Not applicable.

\section{Availability of data and materials}

Data supporting the conclusions of this article are included within the article. The datasets used and/or analyzed during the present study are available from the corresponding author upon reasonable request.

\section{Competing interests}

The authors declare that they have no competing interests.

\section{Funding}

This work was funded by The Royal Patronage of Her Royal Highness Princess Maha Chakri Sirindhorn Walailak University Botanic Garden, under the project entitled: Medicinal under-exploited Thai native plant against Acanthamoeba, Leishmania donovani, and Plasmodium falciparum - Toward South East Asia collaboration initiative (Grant No. 040226)

\section{Authors' contributions}

SS performed all the laboratory work, analyzed the data and wrote a draft of the paper; VN conceived the idea, designed the experiments related to natural products and parasitology, and overlooked at the laboratory work; WM assisted in the experiments; All authors read, reviewed, edited, agreed, and approved for the submission of the finalized manuscript.

\section{Author details}

${ }^{1}$ School of Allied Health Sciences, Southeast Asia Water Team (SEA Water Team) and World Union for Herbal Drug Discovery (WUHeDD), and Research Excellence Center for Innovation and Health Products (RECIHP), Walailak University, Nakhon Si Thammarat, Thailand. ${ }^{2}$ Graduate School, Prince of Songkla University, Songkhla, Thailand. ${ }^{3}$ Department of Pharmacognosy and Pharmaceutical Botany, Faculty of Pharmaceutical Sciences, Chulalongkorn University, Bangkok, Thailand. ${ }^{4}$ Department of Biochemistry, Habib Medical School, Islamic University in Uganda, Kampala, Uganda. ${ }^{5}$ Department of Clinical Tropical Medicine, Faculty of Tropical Medicine, Mahidol University, Bangkok, Thailand. ${ }^{6}$ Department of Biology, Faculty of Sciences, São Paulo State University, São Paulo, Brazil. ${ }^{7}$ Department of Microbiology, National Institute of Tuberculosis \& Respiratory Diseases (NITRD), New Delhi, India. ${ }^{8}$ Department of Medical 
Sciences \& CICECO-Aveiro Institute of Materials, University of Aveiro, Aveiro, Portugal. ${ }^{9}$ Department of Biotechnology \& Genetic Engineering, University of Development Alternative Lalmatia, Dhaka, Bangladesh. ${ }^{10}$ School of Pharmacy, University of Nottingham Malaysia Campus, Selangor, Malaysia.

${ }^{11}$ Ferdows School of Paramedical and Health, Birjand University of Medical Sciences, Birjand, Iran.

\section{References}

1. Chu D, Miles H, Toney D, Ngyuen C, Marciano-Cabral F. Amebicidal activity of plant extracts from Southeast Asia on Acanthamoeba spp. Parasitol Res. 1998;84:746-52.

2. Sifaoui I, Reyes-Batlle M, López-Arencibia A, Wagner C, Chiboub O, Rodríguez JA, et al. Evaluation of the anti-Acanthamoeba activity of two commercial eye drops commonly used to lower eye pressure. Exp Parasitol. 2017;183:117-23.

3. Chao M, Thongseesuksai T, Boonmars T, Laummaunwai P. Investigation of the in vitro cysticidal activity of miltefosine against Acanthamoeba spp. J Parasit Dis. 2020;44:491-5.

4. Walochnik J, Aichelburg A, Assadian O, Steuer A, Visvesvara G, Vetter N, et al. Granulomatous amoebic encephalitis caused by Acanthamoeba amoebae of genotype T2 in a human immunodeficiency virus-negative patient. J Clin Microbiol. 2008;46:338-40.

5. Lorenzo-Morales J, Khan NA, Walochnik J. An update on Acanthamoeba keratitis: diagnosis, pathogenesis and treatment. Parasite. 2015;22:10.

6. Taher M, Tg Zakaria T, Susanti D, Zakaria ZA. Hypoglycaemic activity of ethanolic extract of Garcinia mangostana Linn. in normoglycaemic and streptozotocin-induced diabetic rats. BMC Complement Altern Med. 2016;16:135.

7. Ibrahim MY, Hashim NM, Mariod AAA. a-mangostin from Garcinia mangostana Linn: An updated review of its pharmacological properties. Arabian J Chem. 2016;9:317-29.

8. Pedraza-Chaverri J, Cárdenas-Rodríguez N, Orozco-Ibarra M, Pérez-Rojas JM. Medicinal properties of mangosteen (Garcinia mangostana). Food Chem Toxicol. 2008;46:3227-39.

9. Cantos R, Lee AF. 2017. The ovicidal potential of the peel and seed ethanolic crude extracts of Garcinia mangostana (Mangosteen) against Ascaris suum. Undergraduate Thesis. Archive AEA (Thesis Section) DLSU-Dasmariñas, Philippines.

10. Canton DGC, Jagunap JJS. 2018. Potential anthelmintic activity of Garcinia mangostana (Mangosteen) pericarp, pulp and seed extracts against Ascaris suum ova. Undergraduate Thesis. Archive AEA (Thesis Section) DLSU-Dasmariñas, Philippines.

11. Mitsuwan W, Bunsuwansakul C, Leonard TE, Laohaprapanon S, Hounkong K, Bunluepuech K, et al. Curcuma longa ethanol extract and Curcumin inhibit the growth of Acanthamoeba triangularis trophozoites and cysts isolated from water reservoirs at Walailak University, Thailand. Pathog Glob Health. 2020;114:194-204.

12. Chu DM, Miles H, Toney D, Ngyuen C, Marciano-Cabral F. Amebicidal activity of plant extracts from Southeast Asia on Acanthamoeba spp. Parasitol Res. 1998;84:746-52. 
13. Lorian V. Antibiotics in laboratory medicine. 4th ed. Baltimore: Williams and Wilkins; 1996.

14. Marciano-Cabral F. Cabral. G. Acanthamoeba spp. as agents of disease in humans. Clin Microbiol Rev. 2003;16:273-307.

15. Pussard M. Pons R. Morphologie de la paroi kystique et taxonomie du genre Acanthamoeba (Protozoa, Amoebida). Protistologica. 1977;13:557 - 98.

16. Xuan YH, Chung BS, Hong YC, Kong HH, Hahn TW, Chung DI. Keratitis by Acanthamoeba triangularis: report of cases and characterization of isolates. Korean J Parasitol. 2008;46:157-64.

17. Gutierrez-Orozco F, Failla ML. Biological activities and bioavailability of mangosteen xanthones: a critical review of the current evidence. Nutrients. 2013;5:3163-83.

18. Mahabusarakam W, Kuaha K, Wilairat P, Taylor WC. Prenylated xanthones as potential antiplasmodial substances. Planta Med. 2006;72:912-6.

19. Upegui Y, Robledo SM, Gil Romero JF, Quinones W, Archbold R, Torres F, et al. In vivo antimalarial activity of a-mangostin and the new xanthone $\delta$-mangostin. Phytother Res. 2015;29:1195-201.

20. Ignatushchenko MV, Winter RW, Riscoe M. Xanthones as antimalarial agents: stage specificity. Am J Trop Med Hyg. 2000;62:77-81.

21. Ke H, Morrisey JM, Qu S, Chantarasriwong O, Mather MW, Theodorakis EA, et al. Caged Garcinia Xanthones, a novel chemical scaffold with potent antimalarial activity. Antimicrob Agents Chemother. 2016;61:e01220-16.

22. Lannang AM, Louh GN, Lontsi D, Specht S, Sarite SR, Florke U, et al. Antimalarial compounds from the root bark of Garcinia polyantha Olv. J Antibiot (Tokyo). 2008;61:518-23.

23. Lyles JT, Negrin A, Khan SI, He K, Kennelly EJ. In vitro antiplasmodial activity of benzophenones and xanthones from edible fruits of Garcinia species. Planta Med. 2014;80:676-81.

24. Castro AP, Kawano T, Spelta LEW, de Castro AT, Pereira NA, Couto FFB, et al. In vivo schistosomicidal activity of 7-epiclusianone and its quantification in the plasma of healthy and Schistosoma mansoni infected mice using UPLC-MS/MS. Phytomedicine. 2018;38:66-73.

25. Konziase B. Protective activity of biflavanones from Garcinia kola against Plasmodium infection. J Ethnopharmacol. 2015;172:214-8.

26. Oluwatosin A, Tolulope A, Ayokulehin K, Patricia O, Aderemi K, Catherine F, et al. Antimalarial potential of kolaviron, a biflavonoid from Garcinia kola seeds, against Plasmodium berghei infection in Swiss albino mice. Asian Pac J Trop Med. 2014;7:97-104.

27. Pereira IO, Assis DM, Juliano MA, Cunha RLOR, Barbieri CL, do Sacramento LVS, et al. Natural products from Garcinia brasiliensis as Leishmania protease inhibitors. J Med Food. 2011;14:55762.

28. Koh JJ, Qiu S, Zou H, Lakshminarayanan R, Li J, Zhou X, et al. Rapid bactericidal action of alphamangostin against MRSA as an outcome of membrane targeting. Biochim Biophys Acta. 2013;1828:834-44. 
29. El-Sayed NM, Ismail KA, Ahmed SA, Hetta MH. In vitro amoebicidal activity of ethanol extracts of Arachis hypogaea L., Curcuma longa L. and Pancratium maritimum L. on Acanthamoeba castellanii cysts. Parasitol Res 2012;110: 1985-1992.

30. Siddiqui R, Aqeel Y, Khan NA. The development of drugs against Acanthamoeba infections. Antimicrob Agents Chemother. 2016;60:6441-50.

31. Fakae LB, Stevenson CW, Zhu XQ, Elsheikha HM. In vitro activity of Camellia sinensis (green tea) against trophozoites and cysts of Acanthamoeba castellanii. Int J Parasitol Drugs Drug Resist. 2020;13:59-72.

32. Anwar A, Yi YP, Fatima I, Khan KM, Siddiqui R, Khan NA, et al. Antiamoebic activity of synthetic tetrazoles against Acanthamoeba castellanii belonging to T4 genotype and effects of conjugation with silver nanoparticles. Parasitol Res. 2020;119:1943-54.

33. Cheesman MJ, llanko A, Blonk B, Cock IE. Developing new antimicrobial therapies: are synergistic combinations of plant extracts/compounds with conventional antibiotics the solution? Pharmacogn Rev. 2017;11:57-72.

34. Heredero-Bermejo I, Sánchez-Nieves J, Soliveri J, Gomez R, de la Mata FJ, Copa-Patino JL, et al. In vitro anti-Acanthamoeba synergistic effect of chlorhexidine and cationic carbosilane dendrimers against both trophozoite and cyst forms. Int J Pharm. 2016;509:1-7.

35. Fatimah $\mathrm{H}$, Nakisah MA. Visualiza on the effect of chlorhexidine gluconate, a biocide on Acanthamoeba sp. by electron microscopy. Malays J Microsc. 2013;9:154-9.

36. Elsheikha HM, Siddiqui R, Khan NA. Drug Discovery against Acanthamoeba Infections: Present Knowledge and Unmet Needs. Pathogens. 2020;9:405.

\section{Figures}
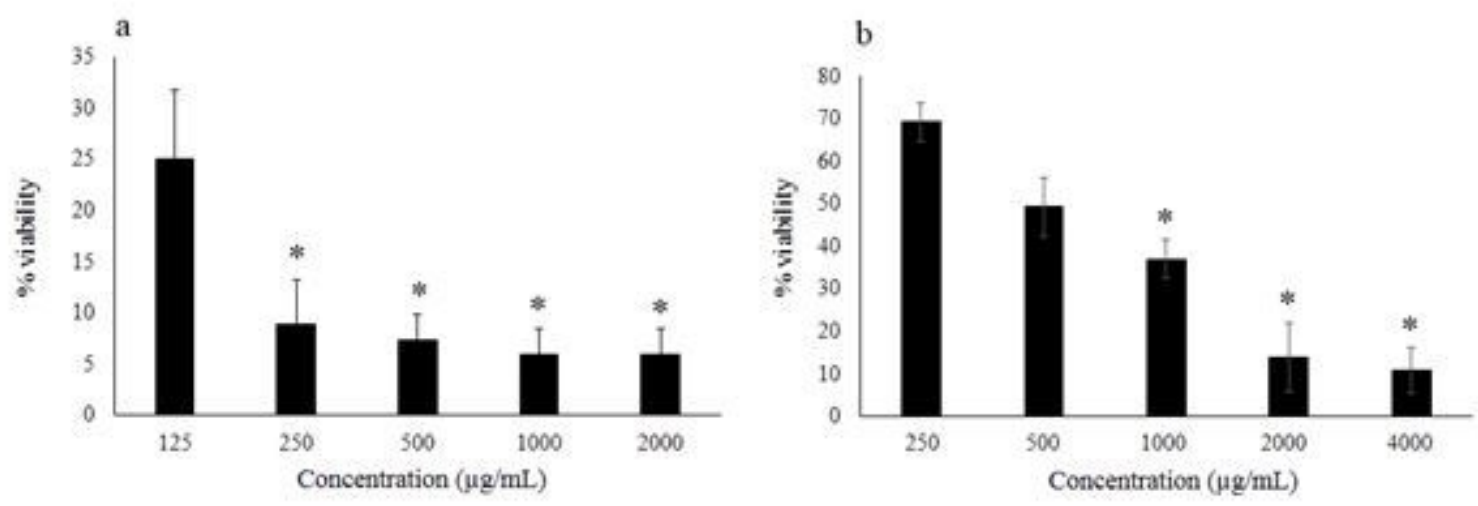

\section{Figure 1}

Effect of G. mangostana extract on viability of A. triangularis trophozoites (a) and cysts (b). Parasite cells were treated with different concentrations of extract, incubated at room temperature for 24 hours. 
Inhibitory activity was carried out using trypan blue exclusion assay. $1 \%$ DMSO was used as negative control. The relative percentange of viability was defined as (mean of the treated /mean of the control) $\times 100$.
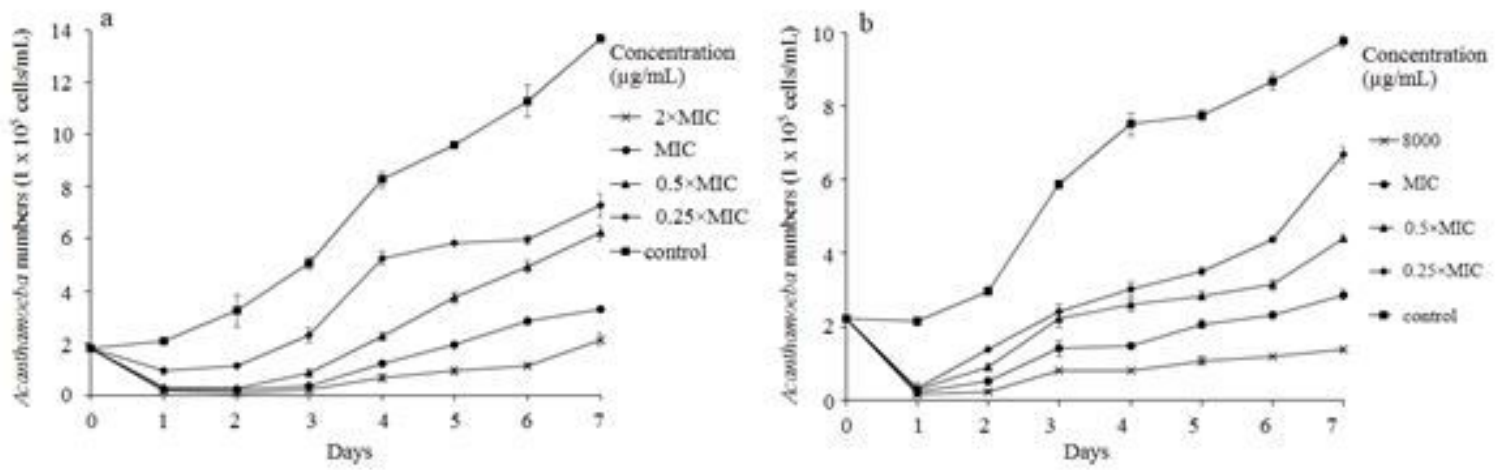

\section{Figure 2}

G. mangostana extract inhibits A. triangularis growth in vitro. To determine the effect of extract on trophozoites (a) and cysts (b), assays were performed by inoculating $2 \times 105$ cells $/ \mathrm{mL}$ in PYG medium in the presence of extract at $2 \times \mathrm{MIC}, \mathrm{MIC}, 0.5 \times \mathrm{MIC}$ and $0.25 \times \mathrm{MIC}$ final concentrations. Inhibitory activity was carried out using trypan blue exclusion assay up to a week. 1\% DMSO was used as negative control. 


\begin{tabular}{|c|c|c|c|c|c|c|}
\hline 7.81 & $9.09 \div 3.14$ & $9.09 \pm 3.14$ & $5.45 \pm 0$ & $5.45 \div 0$ & $5.45 \pm 0$ & 0 \\
\hline 3.90 & $23.63 \div 3.14$ & $40.00 \pm 3.14$ & $36.36 \div 3.14$ & $27.27 \div 0$ & $12.72 \div 3.14$ & 0 \\
\hline 1.95 & $47.27 \div 3.14$ & $52.72 \pm 3.14$ & $45.45 \div 3.14$ & $34.54=3.14$ & $23.63 \pm 3.14$ & 0 \\
\hline 0.97 & $54.54 \div 5.45$ & $54.54 \pm 0$ & $45.45 \div 3.14$ & $40.00 \div 3.14$ & $25.45 \div 3.14$ & $5.45 \pm 0$ \\
\hline 0.48 & $78.18 \div 3.14$ & $74.54 \pm 3.14$ & $60.00 \pm 5.45$ & $47.27 \div 3.14$ & $29.09 \div 3.14$ & $5.45 \pm 0$ \\
\hline 0 & $100 \pm 0$ & $72.72 \pm 6.29$ & $58.18 \div 3.14$ & $47.27 \div 3.14$ & $27.27 \pm 0$ & $12.72 \div 3.14$ \\
\hline & 0 & 15.62 & 31.25 & 62.5 & 125 & 250 \\
\hline
\end{tabular}

Concentration extract $(\mu \mathrm{g} / \mathrm{ml})$



Figure 3

Combination of G. mangostana extract and chlorhexidine for anti-amoebic effects on trophozoites of A. triangularis. Parasites were grown in PYG medium in the presence of extract alone and in combination with chlorhexidine for 24 hours. Inhibitory activity was carried out using trypan blue exclusion assay. $1 \%$ DMSO was used as negative control. The relative percentage of viability was defined as (mean of the treated /mean of the control) $\times 100$. 


\begin{tabular}{|c|c|c|c|c|c|c|}
\hline 62.50 & $7.01 \pm 3.03$ & $5.26 \pm 0$ & $5.26 \pm 0$ & $5.26 \pm 0$ & 0 & 0 \\
\hline 31.25 & $29.82 \div 3.03$ & $22.80=3.03$ & $7.01 \pm 3.03$ & $7.01 \pm 3.03$ & $7.01=3.03$ & 0 \\
\hline 15.62 & $57.89 \pm 0$ & $17.54 \pm 3.03$ & $7.01 \pm 3.03$ & $7.01 \pm 3.03$ & $7.01 \pm 3.03$ & $3.50=3.03$ \\
\hline 7.81 & $87.71=0.67$ & $45.61 \div 3.03$ & $7.01 \pm 3.03$ & $7.01 \div 3.03$ & $7.01 \div 3.03$ & $7.01 \div 3.03$ \\
\hline 3.9 & $96.49 \div 3.03$ & $49.12 \div 3.03$ & $12.28=3.03$ & $14.03 \div 3.03$ & $10.52 \pm 0$ & $10.52 \pm 0$ \\
\hline 0 & $100 \pm 0$ & $59.64 \div 3.03$ & $59.64 \div 3.03$ & $24.56 \div 3.03$ & $7.01 \div 3.03$ & $8.77 \div 3.03$ \\
\hline & 0 & 250 & 500 & 1000 & 2000 & 4000 \\
\hline
\end{tabular}

Concentration extract $(\mu \mathrm{g} / \mathrm{ml})$

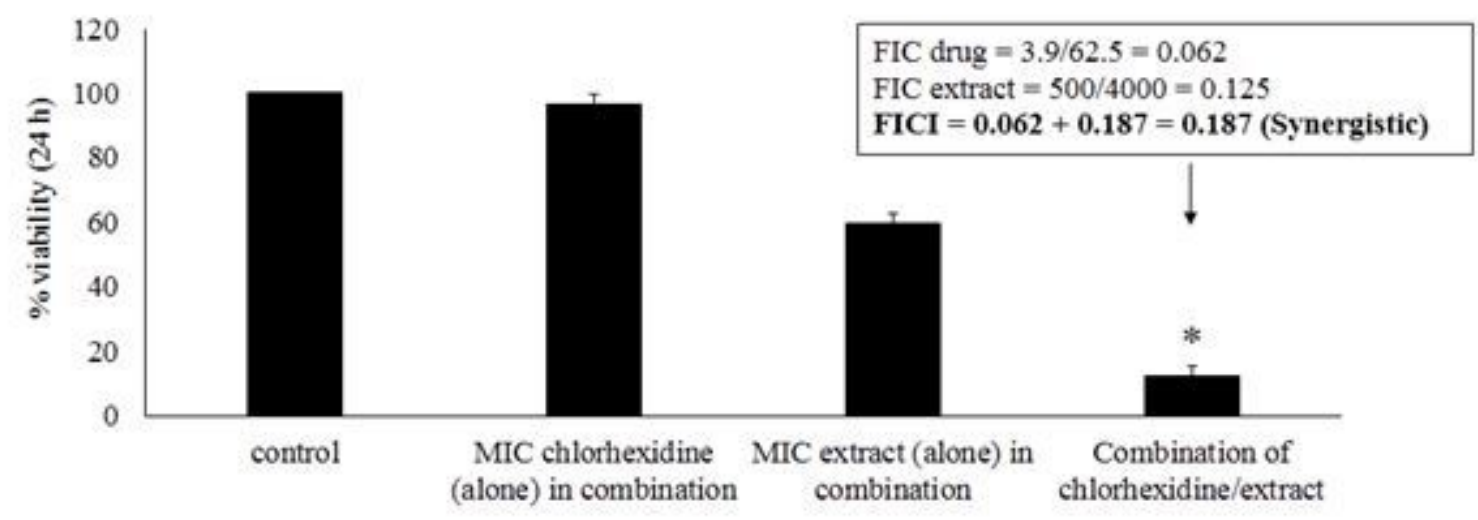

Figure 4

Combination of G. mangostana extract and chlorhexidine for anti-amoebic effect on cysts of A. triangularis. Parasites were grown in PYG medium in the presence of extract alone and in combination with chlorhexidine for 24 hours. Inhibitory activity was carried out using trypan blue exclusion assay. $1 \%$ DMSO was used as negative control. The relative percentage of viability was defined as (mean of the treated /mean of the control) $\times 100$. 


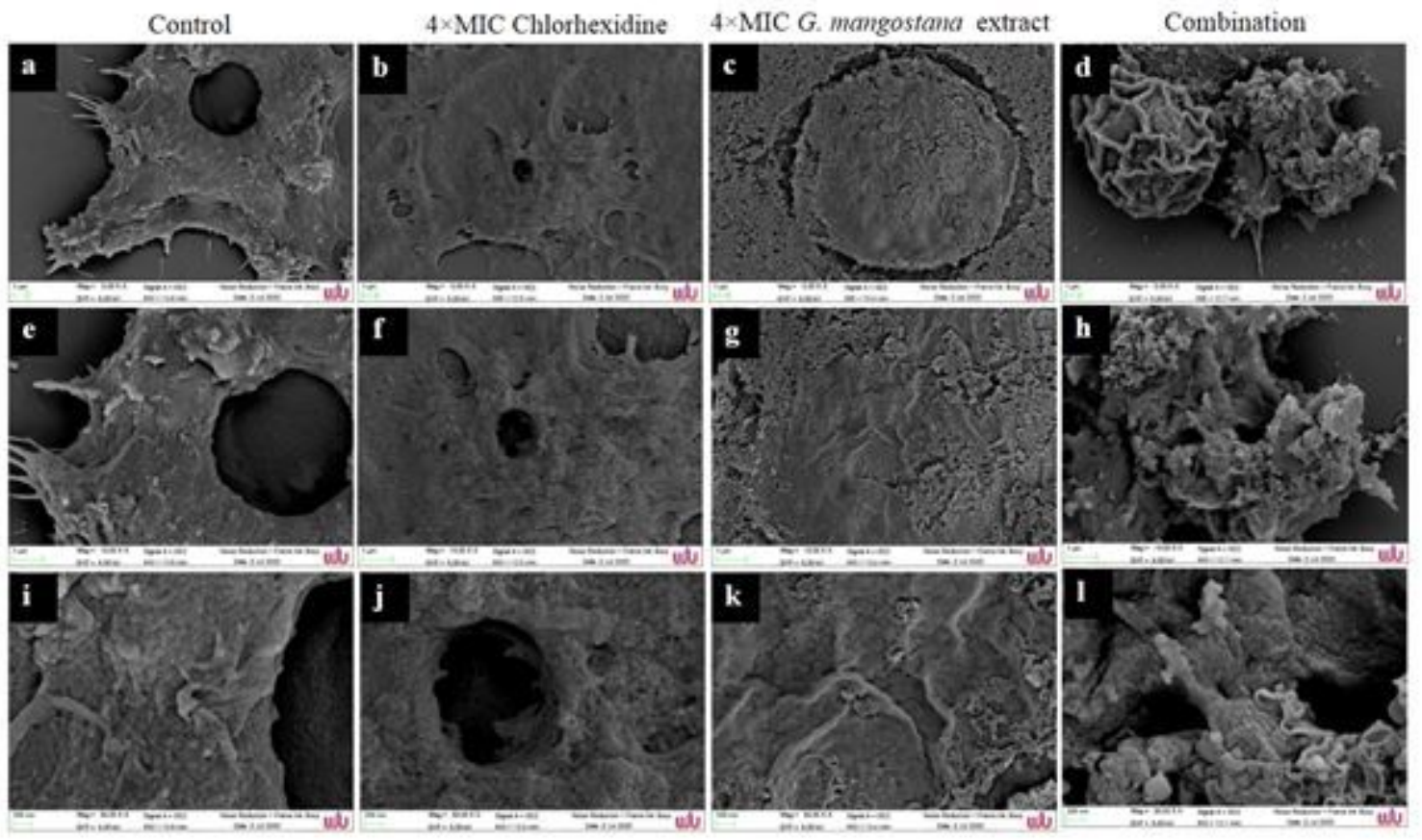

\section{Figure 5}

Scanning micrographs of A. triangularis trophozoites after treatment with G. mangostana extract, chlorhexidine and in combination at 24 hours. Control trophozoites (a,e,i); trophozoites were treated with $4 \times$ MIC chlorhexidine (b,f,j); $4 \times$ MIC G. mangostana extract $(c, g, k)$ and combination (d,h,l), respectively. Magnification: $\mathrm{a}-\mathrm{d}=$ =5000 $; \mathrm{e}-\mathrm{h}=10000 \mathrm{X} ; \mathrm{i}-\mathrm{I}=30000 \mathrm{X}$.

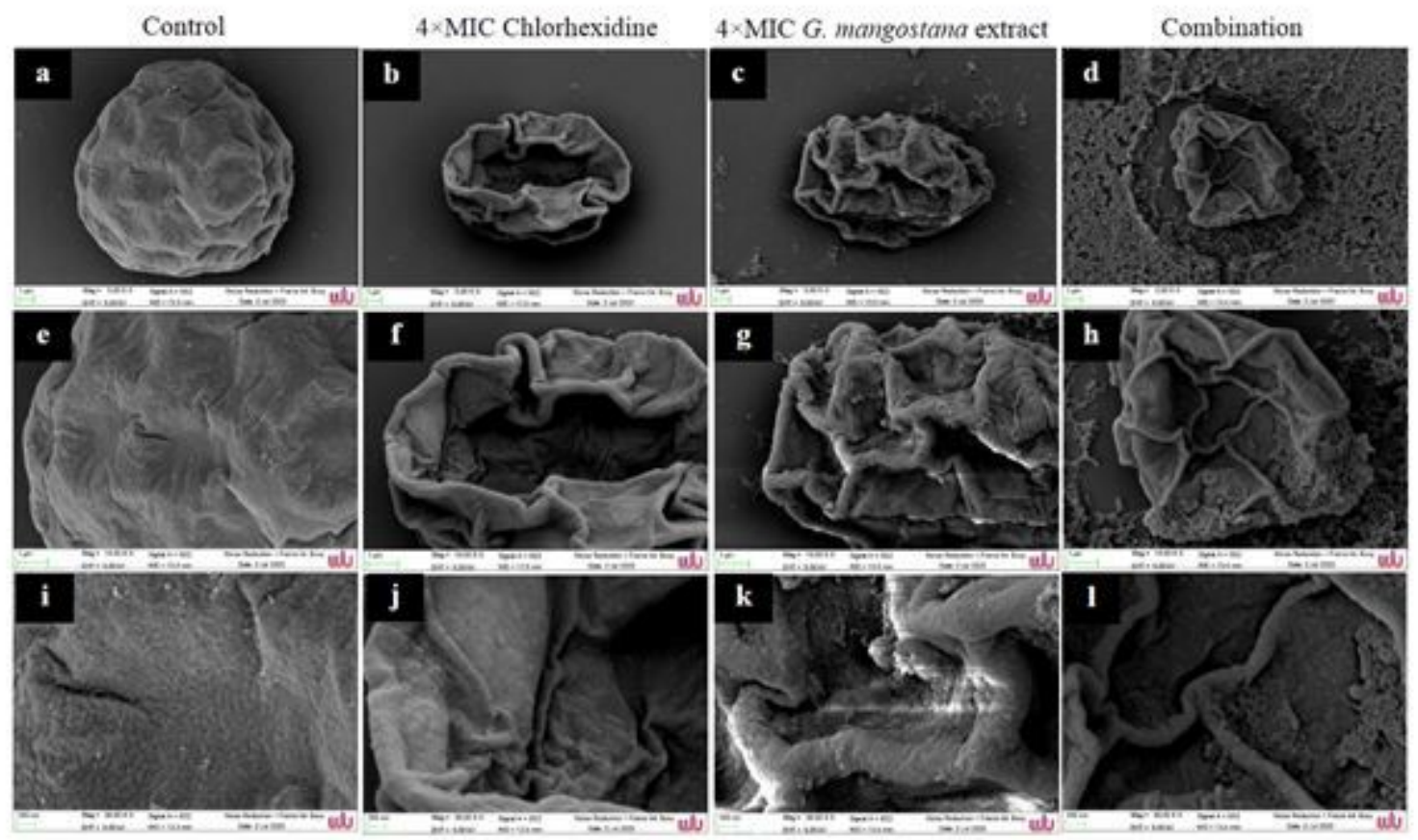

\section{Figure 6}


Scanning micrographs of A. triangularis cysts after treatment with $\mathrm{G}$. mangostana extract, chlorhexidine and in combination at 24 hours. Control cysts $(a, e, i)$; cysts were treated with $4 \times \mathrm{MIC}$ chlorhexidine $(b, f, j)$; $4 \times$ MIC G. mangostana extract $(c, g, k)$ and combination $(d, h, l)$, respectively. Magnification: $a-d=5000 X ;$ e-h $=10000 X ; i-I=30000 X$. 